\title{
Robust flux balance analysis of multiscale biochemical reaction networks
}

\author{
Yuekai Sun ${ }^{*}{ }^{*}$, Ronan MT Fleming ${ }^{2,3}$, Ines Thiele ${ }^{2,3}$ and Michael A Saunders ${ }^{4}$
}

\begin{abstract}
Background: Biological processes such as metabolism, signaling, and macromolecular synthesis can be modeled as large networks of biochemical reactions. Large and comprehensive networks, like integrated networks that represent metabolism and macromolecular synthesis, are inherently multiscale because reaction rates can vary over many orders of magnitude. They require special methods for accurate analysis because naive use of standard optimization systems can produce inaccurate or erroneously infeasible results.

Results: We describe techniques enabling off-the-shelf optimization software to compute accurate solutions to the poorly scaled optimization problems arising from flux balance analysis of multiscale biochemical reaction networks. We implement lifting techniques for flux balance analysis within the openCOBRA toolbox and demonstrate our techniques using the first integrated reconstruction of metabolism and macromolecular synthesis for E. coli.

Conclusion: Our techniques enable accurate flux balance analysis of multiscale networks using off-the-shelf optimization software. Although we describe lifting techniques in the context of flux balance analysis, our methods can be used to handle a variety of optimization problems arising from analysis of multiscale network reconstructions.
\end{abstract}

\section{Background}

Let $S \in \mathbf{R}^{m \times n}$ be a stoichiometric matrix that represents a biochemical network consisting of $m$ species interacting via $n$ reactions. Flux balance analysis (FBA) predicts steady state reaction rates (fluxes) of such a biochemical network by solving the linear program

$$
\begin{array}{cl}
\underset{v}{\operatorname{maximize}} & c^{T} v \\
\text { subject to } & S v=0, \\
& v_{l} \leq v \leq v_{u},
\end{array}
$$

where $v_{l}, v_{u} \in \mathbf{R}^{n}$ are lower and upper bounds on the fluxes and $c$ represents a biologically motivated objective function. We refer to [1] for details about FBA.

Recently, Thiele et al. [2] described the first genomescale integrated reconstruction of $E$. coli metabolism and macromolecular synthesis that represents the function of almost 2000 genes. This Metabolic-Expression model explicitly accounts for the demands of macromolecular synthesis at single nucleotide resolution. To enforce

\footnotetext{
*Correspondence: yuekai@stanford.edu

${ }^{1}$ Institute for Computational and Mathematical Engineering, Stanford University, Stanford, USA

Full list of author information is available at the end of the article
}

consistency between the state of metabolism and macromolecular synthesis, Thiele et al. introduce coupling constraints on certain pairs of fluxes (for example, the fluxes for a metabolic reaction and the reaction responsible for synthesizing the enzyme that catalyzes the metabolic reaction [3]):

$$
c_{\min } \leq \frac{v_{1}}{v_{2}} \leq c_{\max }
$$

where $c_{\min }, c_{\max }>0$. Each coupling constraint can be formulated as a pair of linear inequality constraints, as described later. We predict the steady state reaction rates of such integrated networks by solving the linear program

$$
\begin{array}{ll}
\underset{v}{\operatorname{maximize}} & c^{T} v \\
\text { subject to } & S v=0, \\
& C v \leq d, \\
& v_{l} \leq v \leq v_{u},
\end{array}
$$

where $C v \leq d$ includes constraints equivalent to (2) for many pairs of fluxes.

Given the inherent multiscale nature of integrated reconstructed networks, the constraint matrices of the FBA linear programs (1) and (3) often contain entries that vary over many orders of magnitude. We say that the 
problems are poorly scaled. Conducting FBA for such networks has been unsatisfactory because even state-of-theart linear programming solvers can produce inaccurate (or erroneously infeasible) results. In particular, for the $E$. coli Metabolic-Expression model, applying CPLEX [4] and Gurobi [5] to (3) with default settings (scaling enabled) has produced results with large constraint violations.

\section{Implementation}

\section{Scaling techniques}

In the context of the simplex method for linear programming, the constraints (including bounds) form a polytope in $n$-space. The condition of a basis matrix associated with a vertex of the polytope provides a quantitative measure of either the "sharpness" or the "flatness" of the vertex. Poorly scaled constraints tend to create a polytope with very sharp and/or very flat vertices. To alleviate numerical difficulties for problem (1), linear programming systems typically compute row and column scaling matrices $D_{r} \in$ $\mathbf{R}^{m \times m}$ and $D_{c} \in \mathbf{R}^{n \times n}$ such that the nonzero entries of the scaled constraint matrix $D_{r} S D_{c}$ are of order 1 . Scaling can improve the condition of many bases, but it may be at the expense of making other bases more ill-conditioned (including the optimal basis). For some problems, such as (3), the scaled constraints $D_{r} S D_{c} \bar{v}=0$ may be satisfied accurately by the scaled solution $\bar{v}$, but when the solution is unscaled, $v=D_{c} \bar{v}$ may violate $S v=0$ significantly. We refer to [6] for a comprehensive study of scaling and its effects on the performance of the simplex method.

\section{Lifting techniques}

Lifting techniques are commonly used in optimization to create an efficient representation of a feasible set. By using auxiliary variables to "lift" the feasible set into a higher-dimensional space, they can dramatically reduce the computational expense (e.g., see Albersmeyer and Diehl [7], Gouveira et al. [8]). The canonical application is for efficiently representing the cross-polytope, i.e., the set

$$
\left\{x \in \mathbf{R}^{n}\left|\sum_{i=1}^{n}\right| x_{i} \mid \leq 1\right\} .
$$

To represent this set in $n$-dimensional space requires $2^{n}$ constraints of the form

$$
\pm x_{1} \pm \cdots \pm x_{n} \leq 1
$$

By introducing $n$ new variables $y_{i}$, thereby lifting the set into $2 n$-dimensional space, we can represent the crosspolytope using $2 n+1$ constraints:

$$
\begin{aligned}
-y_{i} & \leq x_{i} \leq y_{i}, \quad i=1, \ldots, n, \\
y_{1}+\cdots+y_{n} & \leq 1 .
\end{aligned}
$$

Here we apply lifting techniques to poorly scaled constraints to make the vertices of the "lifted" polytope more regular. Note that small entries in $S$ and $C$ do not constitute poor scaling unless all entries in a row or column are small. (There are no such rows and columns in our test data, but in general they would be scaled up to have maximum entry 1.) Our explicit aim is to reduce the magnitude of the largest matrix entries so that the reformulated constraints do not need scaling.

\section{Mass balance constraints}

In problem (1), the mass balance constraints $S v=0$ often contain poorly scaled reactions such as

$$
A+10000 B \rightarrow C+D,
$$

which may represent the synthesis of a macromolecule in a reconstruction. We can decompose such reactions into sequences of reactions involving dummy metabolites with reasonably scaled coefficients. For example, (4) is equivalent to two reactions involving a dummy metabolite $\hat{B}$ :

$$
A+100 \hat{B} \rightarrow C+D, \quad 100 B \rightarrow \hat{B} .
$$

\section{Coupling constraints}

In problem (3), the constraints $C x \leq d$ include equivalents of the coupling constraints (2). These enforce consistency between the states of the metabolic and macromolecular synthesis reactions and are often poorly scaled because reaction rates can vary over many orders of magnitude. For example, two fluxes could be related by

$$
0.0001 \leq \frac{v_{1}}{v_{2}} \leq 10000 .
$$

As before, we can decompose these constraints into sequences of constraints involving auxiliary variables with reasonable coefficients. If the second inequality in (6) were presented to our implementation as $v_{1} \leq 10000 v_{2}$, we would transform it to two constraints involving an auxiliary variable $s_{1}$ :

$$
v_{1} \leq 100 s_{1}, \quad s_{1} \leq 100 v_{2} .
$$

If the first inequality in (6) were presented as $v_{1} \geq$ $0.0001 v_{2}$, we would leave it alone, but the equivalent inequality $10000 v_{1} \geq v_{2}$ would be transformed to

$$
v_{2} \leq 100 s_{2}, \quad s_{2} \leq 100 v_{1} .
$$

\section{Hierarchical lifting}

Our implementation of lifting techniques uses a parameter $\tau$, set to 1024 in our experiments. Constraints containing entries larger than $\tau$ are reformulated.

Very large entries might require more than one auxiliary variable and constraint. In these cases, we choose the reformulated constraint coefficients to be equally spaced in logarithmic scale. For example, the poorly scaled reaction

$$
A+10^{9} B \rightarrow C+D
$$


(with $\left|10^{9}\right|>\tau$ ) would be reformulated as

$$
\begin{aligned}
A+1000 B_{1} & \rightarrow C+D, \\
1000 B_{2} & \rightarrow B_{1}, \\
1000 B & \rightarrow B_{2}
\end{aligned}
$$

(with $|1000| \leq \tau$ ).

\section{Comment}

Unlike traditional scaling, the above lifting techniques transform poorly scaled constraints without affecting other constraints. The linear program does become larger (more constraints and variables), but the added constraints are extremely sparse and should have little impact on the performance of a typical large-scale solver (see Figure 1). Indeed, the time per iteration for the simplex method could well decrease because smaller "large" entries in the basis matrices typically lead to sparser basis factorizations.

\section{Iterative refinement}

After a simplex solver has returned an allegedly optimal basic solution, the accuracy of satisfying the general linear constraints $(S v=0$ and $C v \leq d$ in (3)) could be improved by applying a single step of classical iterative refinement [9], especially if extended precision were available. However, the refined basic solution could well lie outside its bounds, and further simplex iterations would be necessary. Ideally this difficulty would be handled by the simplex solver itself.

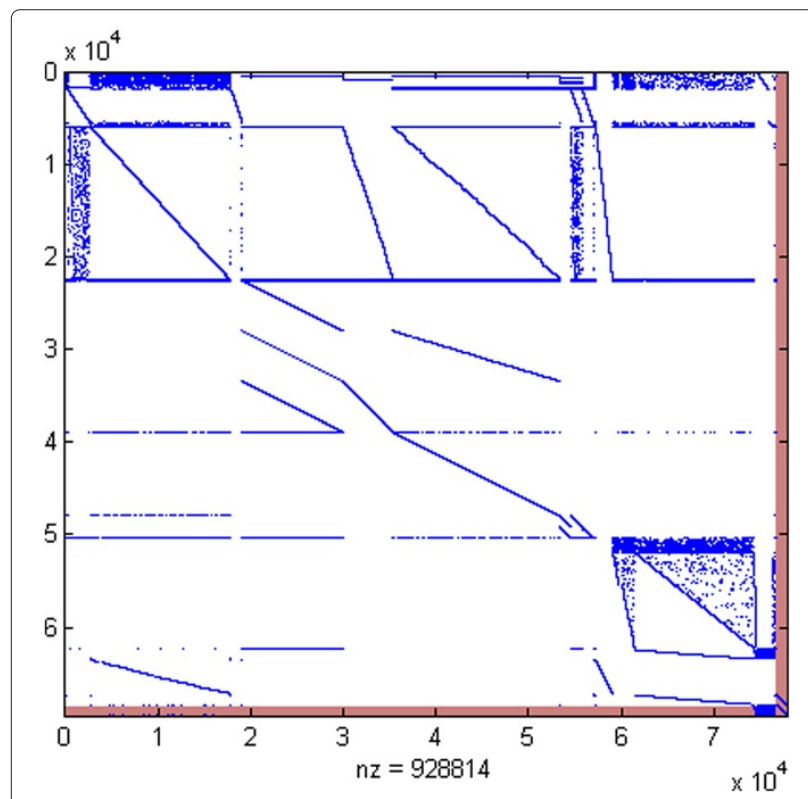

Figure $1 \mathrm{E}$. coli Metabolic-Expression matrix before and after lifting. Spy plot of the E. coli Metabolic-Expression matrix before and after lifting. The red areas were added by the lifting procedure and are very sparse.
We note that more elaborate forms of iterative refinement have been used to improve the accuracy of linear programming solutions. Gleixner et al. [10] describe an incremental precision-boosting procedure that solves a sequence of linear programs, each attempting to correct the error in the previous optimal solution. The Zoom procedure of Saunders and Tenenblat [11] is an analogous strategy for interior methods.

\section{Implementation in the openCOBRA toolbox}

Lifting techniques for poorly scaled reactions and coupling constraints have been implemented in the openCOBRA toolbox 2.05 [12], a Matlab package for constraint-based reconstruction and analysis of biochemical networks. Algorithm 1 summarizes the main steps. Our implementation makes efficient use of auxiliary variables by reusing them if possible. Suppose metabolite $A$ participates in more than one reaction with large stoichiometric coefficients. We can use the same auxiliary variable to decompose all reactions involving metabolite $A$, thereby keeping problem size to a minimum.

To benefit from solving the reformulated problem, we must disable scaling and any "presolve" option that would permit reaggregation of constraints. Our implementation automatically sets these options for CPLEX and Gurobi.

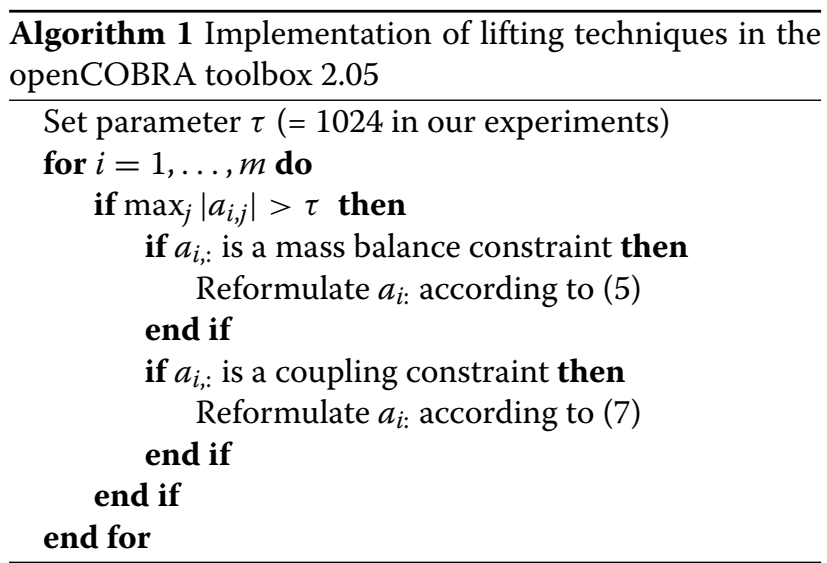

Table 1 FBA results for ME76664 before and after lifting

\begin{tabular}{llllll}
\hline $\mathbf{6 8 2 9 9}$ rows & \multicolumn{2}{l}{ Simplex } & & & Barrier \\
\cline { 2 - 3 } \cline { 5 - 6 } $\mathbf{7 6 6 6 4}$ columns & Before & After & & Before & After \\
\hline Iterations & 48603 & 58288 & & 56490 & 9985 \\
CPU time & 242 & 292 & & 384 & 93 \\
Infeasibilities & $1.3 \times 10^{-4}$ & $2.9 \times 10^{-6}$ & & $1.4 \times 10^{-1}$ & $3.4 \times 10^{-6}$ \\
\hline
\end{tabular}

FBA results for the E. coli Metabolic-Expression model ME76664 using CPLEX primal simplex and barrier solvers. Iterations, time, and sum of infeasibilities before and after lifting. The iterations in columns 4 and 5 include about 100 for the barrier solver and the remainder for the simplex crossover. 
Table 2 FBA results for ME76589 before and after lifting

\begin{tabular}{llllll}
\hline $\mathbf{6 8 2 9 9}$ rows & \multicolumn{2}{l}{ Simplex } & & & Barrier \\
\cline { 2 - 3 } \cline { 5 - 6 } $\mathbf{7 6 5 8 9}$ columns & Before & After & & Before & After \\
\hline Iterations & 22649 & 70786 & & 22816 & 32278 \\
CPU time & 209 & 601 & & 350 & 584 \\
Infeasibilities & $9.7 \times 10^{-4}$ & $9.8 \times 10^{-7}$ & & $7.1 \times 10^{-2}$ & $6.2 \times 10^{-5}$ \\
\hline
\end{tabular}

FBA results for the $E$. coli Metabolic-Expression model ME76589 using CPLEX primal simplex and barrier solvers. Again, most of the barrier iterations are for the simplex crossover.

\section{Results and discussion}

We use our implementation of lifting techniques to conduct FBA on two Metabolic-Expression models of E. coli [2]. The models (ME76664 and ME76589) represent the function of almost $2000 \mathrm{E}$. coli genes and involve 62212 metabolites, with 6087 coupling constraints $C v \leq d$ to enforce consistency between the predicted steady states of both metabolism and macromolecular synthesis. The first model (ME76664) accounts for 76664 reactions, and the second (ME76589) accounts for 76589 reactions. Because of the dependencies between pairs of metabolic reactions and macromolecular synthesis reactions, the resulting flux balanced steady state $v$ has reaction rates that vary by four orders of magnitude [2]. Both models have about 41,000 large matrix entries (exceeding $\tau=1024$ ), with 1825 entries exceeding $10^{5}$ and biggest entry $8 \times 10^{5}$.

Conducting FBA on ME76664 using the CPLEX and Gurobi simplex and barrier solvers with default settings (including scaling) resulted in erroneous reports of infeasibility or "optimal" solutions that were significantly infeasible. Our own simplex solver SQOPT [13] with scaling activated would solve the scaled problem well, but unscaling would magnify the infeasibilities.
Table 3 FVA results (simplex solvers) for ME76664 before and after lifting

\begin{tabular}{rrrrrr}
\hline & \multicolumn{2}{c}{ Iterations } & & \multicolumn{2}{c}{ Infeasibilities } \\
\cline { 2 - 3 } \cline { 6 - 6 } Flux & Before & After & & Before & After \\
\hline 1 & 22510 & 44496 & & $1.1 \times 10^{-4}$ & $7.6 \times 10^{-5}$ \\
5001 & 30405 & 40318 & & $1.5 \times 10^{-4}$ & $9.6 \times 10^{-5}$ \\
10001 & 34963 & 41231 & & $9.4 \times 10^{-2}$ & $8.2 \times 10^{-5}$ \\
15001 & 103210 & 41891 & & $4.5 \times 10^{-5}$ & $9.4 \times 10^{-6}$ \\
20001 & 120089 & 40587 & & $8.8 \times 10^{-2}$ & $8.3 \times 10^{-5}$ \\
25001 & 30786 & 41161 & & $1.7 \times 10^{-4}$ & $8.3 \times 10^{-5}$ \\
30001 & 55177 & 40534 & & $9.8 \times 10^{-2}$ & $8.1 \times 10^{-5}$ \\
35001 & 68760 & 40933 & & $1.3 \times 10^{-4}$ & $8.3 \times 10^{-5}$ \\
40001 & 30360 & 40778 & & $1.2 \times 10^{-4}$ & $1.6 \times 10^{-5}$ \\
45001 & 107485 & 40905 & & $3.1 \times 10^{-5}$ & $8.3 \times 10^{-5}$ \\
50001 & 32553 & 40360 & & $9.7 \times 10^{-2}$ & $8.5 \times 10^{-5}$ \\
55001 & 20661 & 39909 & & $9.5 \times 10^{-5}$ & $5.7 \times 10^{-5}$ \\
60001 & 25477 & 39830 & & $9.4 \times 10^{-2}$ & $8.6 \times 10^{-5}$ \\
65001 & 139251 & 42230 & & $2.9 \times 10^{-5}$ & $8.4 \times 10^{-5}$ \\
70001 & 137611 & 42389 & $6.2 \times 10^{-5}$ & $8.3 \times 10^{-5}$ \\
75001 & 40930 & 41139 & & $4.0 \times 10^{-5}$ & $8.2 \times 10^{-5}$ \\
\hline & & & & &
\end{tabular}

FVA results for the E. coli Metabolic-Expression model ME76664 using the CPLEX simplex solvers. Iterations and sum of infeasibilities for dual simplex (default) before lifting and for primal simplex after lifting. The first column lists which variable is being maximized. Lifting helps the CPLEX simplex solvers.

With the CPLEX solvers, our lifting techniques eliminate infeasible reports and significantly reduce the infeasibility of the computed steady states; see Table 1 and Table 2. Note that most of the "barrier iterations" are really simplex iterations required by crossover (the procedure for

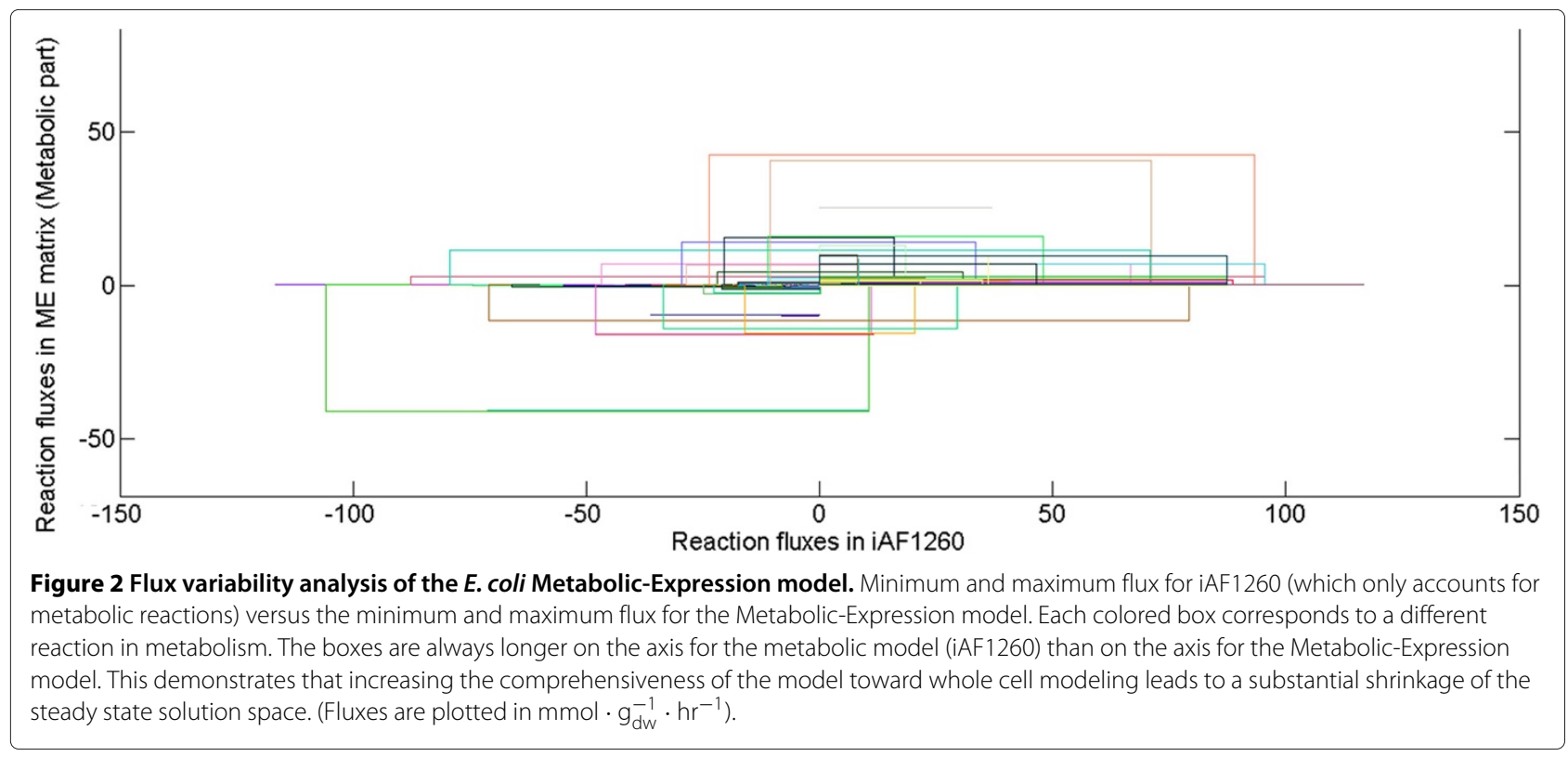


Table 4 FVA results (barrier solver) for ME76664 before and after lifting

\begin{tabular}{|c|c|c|c|c|}
\hline \multirow[b]{2}{*}{ Flux } & \multicolumn{2}{|c|}{ Iterations } & \multicolumn{2}{|c|}{ Infeasibilities } \\
\hline & Before & After & Before & After \\
\hline 1 & 76669 & 23084 & $9.4 \times 10^{-2}$ & $9.0 \times 10^{-2}$ \\
\hline 5001 & 34721 & 66731 & $4.7 \times 10^{0}$ & $8.8 \times 10^{-2}$ \\
\hline 10001 & 58672 & 85819 & $9.3 \times 10^{-2}$ & $9.3 \times 10^{-4}$ \\
\hline 15001 & 28032 & 47901 & $9.7 \times 10^{-2}$ & $1.6 \times 10^{-2}$ \\
\hline 20001 & 18715 & 30433 & $3.2 \times 10^{-2}$ & $3.0 \times 10^{-2}$ \\
\hline 25001 & 12224 & 17973 & $3.5 \times 10^{2}$ & $8.8 \times 10^{-2}$ \\
\hline 30001 & 19621 & 35111 & $8.8 \times 10^{-2}$ & $3.1 \times 10^{-2}$ \\
\hline 35001 & 6743 & 7630 & $1.0 \times 10^{-2}$ & $6.8 \times 10^{-2}$ \\
\hline 40001 & 47609 & 4111 & $8.8 \times 10^{-2}$ & $9.4 \times 10^{-2}$ \\
\hline 45001 & 9117 & 9980 & $1.0 \times 10^{-1}$ & $3.2 \times 10^{-2}$ \\
\hline 50001 & 9567 & 49350 & $9.5 \times 10^{-2}$ & $9.5 \times 10^{-2}$ \\
\hline 55001 & 23985 & 13362 & $9.6 \times 10^{-2}$ & $1.1 \times 10^{-1}$ \\
\hline 60001 & 99067 & 27075 & $1.1 \times 10^{-1}$ & $9.1 \times 10^{-2}$ \\
\hline 65001 & 44796 & 11509 & $3.1 \times 10^{-1}$ & $8.9 \times 10^{-2}$ \\
\hline 70001 & 17045 & 14393 & $4.0 \times 10^{-2}$ & $6.5 \times 10^{-2}$ \\
\hline 75001 & 20790 & 14908 & $9.0 \times 10^{-2}$ & $5.6 \times 10^{-6}$ \\
\hline
\end{tabular}

FVA results for the E. coli Metabolic-Expression model ME76664 using the CPLEX barrier solver. Iterations and sum of infeasibilities before and after lifting. The first column lists which variable is being maximized. Columns 2 and 3 list the total iterations for the barrier solver and the simplex crossover (with barrie requiring only about 100 iterations in all cases). CPLEX barrier appears less reliable than the CPLEX simplex solvers on this model.

finding a basic solution from the barrier solution). These do not alter the optimal objective value and may not be essential in practice.

We also used lifting to conduct flux variability analysis (FVA) [14] for the ME76664 model and obtained biologically consistent results (see Figure 2). We compared the flux span of each metabolic reaction in ME76664 with the flux span of the corresponding reaction in the E. coli metabolic model (iAF1260) [15]. The chief difference between these two models is that in ME76664 the metabolic building blocks (e.g., amino acids) are used to synthesize the metabolic enzymes, which in turn catalyze the metabolic reactions, while in iAF1260 the building blocks are collected in a static biomass reaction. Artifacts with FBA on metabolic models, such as thermodynamically infeasible flux around stoichiometrically balanced reaction cycles, are eliminated for all enzymecatalyzed reactions in ME76664, as the coupling constraints penalize high flux rates. These constraints also restrict the maximum possible flux rates through enzyme catalyzed reactions due to the demand-supply challenge for the building blocks, thus limiting the set of possible transcriptomes and proteomes of the model. Overall, the feasible steady state solution space is substantially reduced in ME76664 compared to the metabolic model alone.
Tables 3 and 4 summarize 15 FVA runs using the CPLEX simplex and barrier solvers. For the simplex method (Table 3) we see that lifting reduces the infeasibilities of the computed steady states and also stabilizes the number of simplex iterations. For the barrier method (Table 4) the effects of lifting are much more varied. The feasibility of the computed steady state is sometimes improved but the lifted problem can take much longer to solve. Evidently the CPLEX barrier solver (with crossover) does not perform reliably on ME76664 with or without lifting.

\section{Conclusions}

We described techniques that enable off-the-shelf optimization software to be applied to multiscale network reconstructions, such as integrated networks that represent both metabolism and macromolecular synthesis. The techniques enable accurate FBA and FVA of an integrated model of metabolism and macromolecular synthesis in $E$. coli, previously impossible because of numerical difficulties encountered by solvers.

As in silico biologists create increasingly complex models that capture more of the multiscale nature of biological systems [16], the optimization problems that arise during the analysis of these models will also become increasingly poorly scaled. We are aware of researchers resorting to specialized packages such as [17] that rely upon rational arithmetic to obtain exact solutions to the FBA and FVA linear programs. Such solvers are likely to be prohibitively slow for analyzing larger, more comprehensive reconstructed networks. A more practical approach is to employ quadruple-precision arithmetic, which is increasingly available in Fortran and $\mathrm{C}$ compilers and is valuable even when implemented in software. In the meantime, our techniques enable the constraint-based modeling community to analyze increasingly sophisticated and comprehensive models of biological systems with improved efficiency and reliability. They could also be combined with the refinement approach of Gleixner et al. [10].

\section{Availability and requirements}

Lifting techniques for poorly scaled reactions and coupling constraints have been implemented in the openCOBRA toolbox 2.05 [12], a MATLAB package for constraint-based reconstruction and analysis of biochemical networks.

Project name: openCOBRA toolbox

Project home page: http://opencobra.sourceforge.net/

Operating system: platform independent

Programming language: $M A T L A B$

Other requirements: MATLAB 2008a or higher

License: GNU GPLv3

Any restrictions to use by non-academics: A separate license must be acquired. 


\section{Competing interests}

The authors declare that they have no competing interests.

\section{Authors' contributions}

YS developed and implemented the lifting techniques. YS and MAS wrote the manuscript. RMTF and IT provided examples, interpreted results, and edited the manuscript. All authors read and approved the final manuscript.

\section{Acknowledgements}

We are grateful to three referees for their insightful comments and suggestions. This work was supported by the Department of Energy (Offices of Advanced Scientific Computing Research and Biological and Environmental Research) as part of the Scientific Discovery Through Advanced Computing program, grant DE-FG02-09ER25917, by the National Institute of General Medical Sciences of the National Institutes of Health, award number U01GM102098, and by the Office of Naval Research, grant N00014-11-1-0067. The content is solely the responsibility of the authors and does not necessarily represent the official views of $\mathrm{DOE}, \mathrm{NIH}$, or ONR.

\section{Author details}

${ }^{1}$ Institute for Computational and Mathematical Engineering, Stanford University, Stanford, USA. ${ }^{2}$ Center for Systems Biology, University of Iceland, Reykjavik, Iceland. ${ }^{3}$ Luxembourg Centre for Systems Biomedicine, University of Luxembourg, Campus Belval, Esch-sur-Alzette, Luxembourg. ${ }^{4}$ Department of Management Science and Engineering, Stanford University, Stanford, USA.

Received: 16 September 2012 Accepted: 8 July 2013

Published: 30 July 2013

\section{References}

1. Orth JD, Thiele I, Palsson $B \varnothing$ : What is flux balance analysis? Nat Biotechnol 2010, 28(3):245-248.

2. Thiele I, Fleming RMT, Que R, Bordbar A, Diep D, Palsson BØ: Multiscale modeling of metabolism and macromolecular synthesis in $E$. coli and its application to the evolution of codon usage. PLOS One 2012, 7(9):e45635. [doi:10.1371/journal.pone.0045635]

3. Thiele I, Fleming RMT, Bordbar A, Schellenberger J, Palsson B $\varnothing$ Functional characterization of alternate optimal solutions of Escherichia coli's transcriptional and translational machinery. Biophys J 2010, 98(10):2072-2081.

4. CPLEX mathematical programming solver [http://www-01.ibm.com/ software/integration/optimization/cplex-optimizer/]

5. Gurobi mathematical programming solver [http://www.gurobi.com/]

6. Elble J, Sahinidis N: Scaling linear optimization problems prior to application of the simplex method. Comput Optimization Appl 2012 52:345-371.

7. Albersmeyer J, Diehl M: The lifted Newton method and its application in optimization. SIAM J Optim 2010, 20(3):1655-1684.

8. Gouveia J, Parrilo PA, Thomas R: Lifts of convex sets and cone factorizations. ArXiv:1111.3164 2011

9. Moler CB: Iterative refinement in floating point. J ACM 1967, 14(2):316-321.

10. Gleixner A, Steffy D, Wolter K: Improving the accuracy of linear programming solvers with iterative refinement. ZIB-Report 12-19, Zuse Institute Berlin 2012

11. Saunders MA, Tenenblat $L$ : The Zoom strategy for accelerating and warm-starting interior methods. Presented at INFORMS Annual Meeting, Pittsburgh, PA Nov 5-8, 2006. [http://www.stanford.edu/group/SOL/talks/ saunders-tenenblat-INFORMS2006.pdf]

12. Schellenberger J, Que R, Fleming RMT, Thiele I, Orth JD, Feist AM, Zielinski DC, Bordbar A, Lewis NE, Rahmanian S, et al.: Quantitative prediction of cellular metabolism with constraint-based models: the COBRA Toolbox v2.0. Nature Protoc 2011, 6(9):1290-1307. [http://github.com/ opencobra]

13. Gill PE, Murray W, Saunders MA: SNOPT: An SQP algorithm for large-scale constrained optimization. SIAM Review 2005, 47:99-131. [SIGEST article].

14. Savinell JM, Palsson BØ: Network analysis of intermediary metabolism using linear optimization. I. Development of mathematical formalism. J Theor Biol 1992, 154(4):421-454.
15. Feist A, Henry C, Reed J, Krummenacker M, Joyce A, Karp P, Broadbelt L, Hatzimanikatis $V$, Palsson $B \varnothing$ : A genome-scale metabolic reconstruction for Escherichia coli K-12 MG1655 that accounts for 1260 ORFs and thermodynamic information. Mol Syst Biol 2007, 3:1-18.

16. Thiele I, Heinken A, Fleming RMT: A systems biology approach to studying the role of microbes in human health. Curr Opin Biotechnol 2012, 21(1):4-12.

17. Cook W, Koch T, Daniel E, Wolter K: An exact rational mixed-integer programming solver. In Proceedings of the 15th international conference on Integer Programming and Combinatorial Optimization, IPCO'11. Berlin, Heidelberg: Springer-Verlag; 2011:104-116. [http://dl.acm.org/citation. cfm?id=2018167]

doi:10.1186/1471-2105-14-240

Cite this article as: Sun et al:: Robust flux balance analysis of multiscale biochemical reaction networks. BMC Bioinformatics 2013 14:240.

\section{Submit your next manuscript to BioMed Central} and take full advantage of:

- Convenient online submission

- Thorough peer review

- No space constraints or color figure charges

- Immediate publication on acceptance

- Inclusion in PubMed, CAS, Scopus and Google Scholar

- Research which is freely available for redistribution

Submit your manuscript at www.biomedcentral.com/submit
C Biomed Central 